\title{
An Ontology-Based Approach to Parsing Turkish Sentences ${ }^{\star}$
}

\author{
Murat Temizsoy and Ilyas Cicekli \\ Dept. of Comp. Eng. and Info. Sc., Bilkent University, \\ 06533 Bilkent, Ankara, TURKEY, \\ \{temizsoy, ilyas\}@cs.bilkent.edu.tr
}

\begin{abstract}
The main problem with natural language analysis is the ambiguity found in various levels of linguistic information. Syntactic analysis with word senses is frequently not enough to resolve all ambiguities found in a sentence. Although natural languages are highly connected to the real world knowledge, most of the parsing architectures do not make use of it effectively. In this paper, a new methodology is proposed for analyzing Turkish sentences which is heavily based on the constraints in the ontology. The methodology also makes use of morphological marks of Turkish which generally denote semantic properties. Analysis aims to find the propositional structure of the input utterance without constructing a deep syntactic tree, instead it utilizes a weak interaction between syntax and semantics. The architecture constructs a specific meaning representation on top of the analyzed propositional structure.
\end{abstract}

\section{Introduction}

One of the main goals of natural language analysis $(N L A)$ is to represent the meaning resides in forms of linguistic usage (spoken or written). The general architecture that is utilized in the current art of computational linguistics is based on a layered approach in which structural (morphological and syntactic) analysis is performed without any interaction with information about word senses and semantics. After parsing the structurally-correct analyses of the input sentence, the information attached with semantics is applied to select one among many. Generally knowledge about the real world or the context is not used, and this causes a real problem in disambiguation [8].

Turkish is a free word-order language, and this makes the analysis task even more complicated. Its flexibility in the sentence structure is a result of morphological inflections (suffixation), and their usage generally provide information about semantics (thematic structure of the sentence, tense and aspect of an event, modality, etc.). This information can be utilized in analyzing the propositional structure of a sentence without dealing with the syntactic constituents like subject, direct object, etc. in general. Motivated with this observation, a new

\footnotetext{
* This research has been supported in part by NATO Science for Stability Program Grant TU-LANGUAGE.
} 
approach for parsing Turkish sentences is presented in this paper. It is heavily based on an rich ontology 15, a knowledge resource to represent entities, events, and the relationships between them in a hierarchical structure. The proposed method tries to find the argument structures of predicates, using morphological information and word-order constraints, and successful analyses are reprocessed to construct the possible meaning representations [26]. Syntax is treated as a formalism to propose analysis for noun phrases (denoting world entities), and ontology-based search decides on the acceptance or rejection of those analyses. So, the method never constructs a complete syntactic tree structure, instead uses ontological constraints.

To present the new methodology proposed in this paper, first we introduce some motivations about why such an approach can be utilized in dealing with difficulties arose from the lack of real world knowledge or the context in Section 2. Then, we briefly describe the structure and the knowledge content of the ontology, and the meaning representation which is the output of the implemented system in Section 3. In that section, we also consider the content of the lexicon and its relationship with the ontology. In Section 4, we describe the proposed methodology which is composed of two components: determination of argument structure and concepts found in the input sentence, and construction of the interlingua which is a rule-based constraint-reasoning module. In the last section, we present the conclusion about the described work together with some possible future extensions.

\section{Motivation}

When we consider the language as a media to exchange information about the real (or some possible) world, the role of a language's structural properties can be reinterpreted as a tool to ease the burden of comprehension (semantic disambiguation). For example, in the sentence "John bought a present for Mary", both entities 'John' and 'Mary' can be the agent of the event 'buy', and it is the syntax that imposes the only interpretation that 'John' is the agent and 'Mary' is the goal. But, language is a phenomenon that cannot be just explained by its structure; it has a close relationship with the real world. There are cases where syntax, even formal semantics, cannot help us to choose one interpretation among many, and it is our real world knowledge that is utilized in selection (at least in preference). For example, consider the following Turkish sentences;

- "Adam kitap okudu"

- "iki kolsuz adam"

In the first sentence, the word 'adam' has two morphological analyses: "the man" and "my island", and both analyses result in syntactically correct sentences. But, only the interpretation "the man read a book" is valid since the event denoted by 'oku' ('read') can only accept a human as an agent, and this eliminates the other interpretation ("my island"). In the second sentence, there are two ways to bracket the noun phrase: [iki [kolsuz adam]] ("two men without 
hands") and [[iki kolsuz] adam] (semantically ill-defined interpretation, since it requires both the existence and non-existence of arms). But, when we consider a simpler phrase "üç kollu adam", although we cannot safely avoid the interpretation "three men with $\operatorname{arm}(\mathrm{s})$ ", we generally prefer the other interpretation, "a man with three arms", since "man with arm" is not informative. Note that, in this sentence, the second bracketing is preferred which is not the case for the first one.

Even with these examples, our power in comprehending language is a result of our knowledge about the real world. In fact, we can reach the same conclusion from another perspective which depends on psycholinguistic observations about human performance on linguistic inputs. Although ungrammatical sentences are common in daily speech contexts (unfinished sentences, improper clause embedding, etc.), information loss in such sentences is minimum, if not zero in most of the circumstances. Even the loss of an utterance segment does not generally affect the comprehension (simultaneous speech in groups, sudden noises, etc.). Also, the effect of context and real world knowledge on the seemingly syntactic phenomenon (Garden Path effect) 10] is demonstrated in psycholinguistic experiments. The effect of context can be seen in the use of Turkish adjectives as denoting an individual entity in the real world.

\section{- "Küçük kırmızı top gittikçe hızlandı"}

In the sentence above, there are four possible interpretations if we are only concerned with the syntactic correctness, and two of them are "the little red thing accelerated as the ball kept going" and "the little red ball accelerated gradually" (note the radical change in interpretation). The first interpretation is possible only if there is a previously mentioned entity that satisfies various constraints (like to be little, to be red, to be in motion, etc.). In all other cases, the second interpretation is preferred, including the null context. But, if those constraints are satisfied, the first interpretation becomes also plausible which means that we have a representation about previously mentioned entities. So, if we are able to represent encountered entities in a sequence of utterances, we can decide which of the interpretations is valid when sentences like the sentence above are uttered.

Started out with these examples in mind, we reach the conclusion that any architecture that is developed for analyzing NL inputs should interact with a representation of the real world, an ontology, and a representation of the context (the entities and the events encountered so far). Since Turkish has an inflectional morphology [9] that provides information about semantic properties, we think that we can analyze Turkish sentences without constructing their corresponding syntactic tree structure. Syntax is used to provide possible analyses for noun and verbal phrases and to limit semantic interpretations according to word-order constraints. It is the thematic structure of a sentence that is to be found through utilizing word senses defined in the lexicon and the representations of entities and events taken from the ontology. In other words, we propose a new methodology in which there is a weak interaction between syntax and 
semantics. In addition, there is also an interaction between syntax and context which represents previously encountered entities and events.

\section{Ontology and Meaning Representation}

Ontology [1511] is the knowledge resource that provides the common sense representation of the real world. It is both utilized in constraining the possible interpretations of a sentence and representing the entities and the events encountered in the context. It is built upon proposed abstractions, concepts, about entities and events. Note that there is a major distinction between entities (atemporal individuals) and events (temporal phenomena). Concepts denoting entities are defined through a set of features with their value-domains, and those features represent the common sense properties of a group of objects. For example, the concept $H U M A N$ has features like name, age, gender, occupation, etc. Some features are given default values (arm-number $=2)$ to make preferences like the ones mentioned in the previous section. An event-concept describes the argument structure (with thematic classifications and constraints on those arguments), the temporal properties, and additional features like entity-concepts. For example, the concept $R E A D$ has arguments agent (limited to $H U M A N$ ) and source (limited to $R E A D A B L E$ ), and its temporal property is durative.

Utilized ontology is not just a set of concepts, it defines a highly-connected network among the concepts. The basic connection among concepts is the relationship between an event-concept and its argument entity-concepts. Note that, each event predicates over a limited set of individuals and it is given as the value-domains of its thematic arguments. Ontology also resembles the hierarchical interpretation of the real world through $i s$ - $a$ relation which defines an inheritance mechanism among concepts. Children concepts define additional features with limitations on the abstraction provided by a parent concept. For example, $H U M A N$ is a $M A M M A L$, which is an $A N I M A L$, etc. So, the previously given features age and gender of $H U M A N$ is in fact provided by $A N I M A L$. There are also other relations which provide extra interpretations about the real world. For example, the relation between an INSTITUTE and a HUMAN (boss-of, member-of, student-of, etc.), or the relation between a MONITOR and a COMPUTER (is-part-of) is also defined in an ontology.

As mentioned, ontology is mainly utilized in finding the relations between entities and events. This is extremely useful in comprehending the correct word sense and eliminating some syntactically correct analyses [3]. For example, consider the following three Turkish sentences:

- "John'dan bir mektup aldım" ("I received a letter from John")

- "Arkadaşımdan bir kalem aldım" ("I took a pencil from my friend")

- "Marketten bir kalem aldım" ("I bought a pencil from the market")

In each sentence, the word 'aldım' is used in three different senses, namely $R E C E I V E, T A K E$, and $B U Y$. Note that, each sentence has the same syntax, an $N P$ with ablative case (denoting the source), an NP with nominative case 
(denoting the theme), and the VP. So, it is impossible to get the correct sense from the syntax. But, if we have constraints on source and theme (such as the source of BUY must be SELLER - COMPANY, and theme of RECEIVE cannot be in the same location with agent, which is a contextual information), then it is easy to eliminate the other interpretations. Similar to this example, the constraint of $R E A D$ (its agent must be a $H U M A N$ ) eliminates the second interpretation of 'adam' ("my island") in the example given in the previous section.

The main purpose of this paper is to analyze Turkish sentences and to represent their intended meaning in an artificial language. So, we need a meaning representation formalism, and we utilize the interlingua representation developed for Microcosmos project in New Mexico State University, called Text Meaning Representation (TMR) 26/11|12]. TMR language is a formalism to represent the relations between events and entities, the semantic properties (aspect, modality, temporal relations, etc.), and pragmatic properties (speech-act, focus, stylistics, etc.). It is heavily based on the ontology, and its propositional content is represented with the concepts from the ontology. It is a frame-based language, and it instantiates the features of the concepts to denote real individuals or events. It may contain several additional frames, besides concepts, to represent other semantic and pragmatic properties. TMR is a suitable language for the purposes of this paper since it provides a mechanism to represent the thematic structure of a sentence.

\section{Methodology}

The computational architecture is based on finding the relationships between the entities (as NPs) and the events (as VPs) of an input sentence, and these relationships are known as the argument structure of the sentence. To achieve this goal, constraints on events' thematic structures and their value-domains, which are defined in the ontology, are used. In other words, the ontology is the major knowledge resource that guides the analysis. In addition, information about morphological markings and constituent order (although Turkish is a free word-order language, it has some limitations in embedded predicates) are utilized in the analysis. NP analysis is generally achieved by the classical $C F G$ formalism. The TMR structure of an input sentence is constructed after its propositional structure is determined. The computational architecture can be analyzed in three distinct components:

1. Morphological Analysis: This phase produces morphologically correct analyses of Turkish words. It is specially important for the current methodology since thematic role of each constituent is explicitly marked in Turkish, and this information is utilized in finding the propositional structure. Also, most of the semantic properties (like tense, aspect, modality, etc.) are explicitly coded through suffixation, and these marks are used in TMR construction. 
2. Semantic Analysis: This phase is the core of the proposed methodology, and it analyzes the propositional structure of the input sentence. It utilizes two knowledge resources, namely the lexicon and the ontology. Its computation is based on a weak interaction between syntax and semantics: syntax proposes NP analyses and puts constituent order constraints, and semantics decides on the acceptance or the rejection of the proposed analysis using knowledge from the ontology.

3. TMR Construction: This phase reanalyzes the constructed propositional structure, uses unprocessed morphological markings, and produces the corresponding TMR representation through using map-rules which are relational constraints between TMR and Turkish [11,12. The core definitions of word senses (without any modifications) are taken from the lexicon where each word sense is defined as a concept instance.

Since morphological analysis is a well known topic with satisfactory computational models, how morphological analysis of Turkish is achieved is not explained here. Morphological analyses of Turkish words are directly taken from an engine developed by Oflazer 9. In the rest of this section, semantic analysis which is the core of this paper is explained in detail, and TMR construction methodology is presented with some examples.

\subsection{Semantic Analysis}

In order to simplify the presentation, first the core idea of the methodology is presented through demonstrating the analysis of a simple Turkish sentence. Let us reconsider the sentence "adam kitap okudu" with the following morphological analysis:

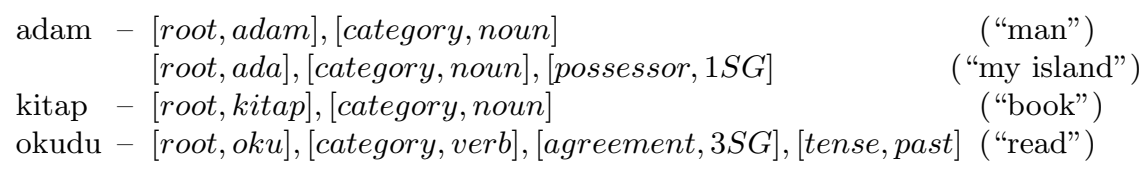

Together with the information from the ontology and the lexicon:

$R E A D$

$\begin{array}{ll}\text { agent } & H U M A N \\ \text { theme } & R E A D A B L E\end{array}$

$\begin{array}{lll}\text { adam } & \stackrel{i s-a}{\longrightarrow} & \text { HUMAN } \\ \text { ada } & \stackrel{i s-a}{\longrightarrow} & \text { LOCATION } \\ \text { kitap } & \stackrel{i s-a}{\longrightarrow} & \text { BOOK } \stackrel{\text { is-a }}{\longrightarrow} \text { READABLE } \\ \text { oku } & \stackrel{i s-a}{\longrightarrow} & \text { READ }\end{array}$

Only the proposition $R E A D(\operatorname{agent}(\operatorname{adam})$, theme(kitap)) is plausible because of the constraints of the arguments of $R E A D$. Note that, this analysis can be achieved without intervening with Turkish syntax, and 'ada' can be directly eliminated since it cannot be an argument of $R E A D$. In Turkish, constituents can change their position rather freely. For example, "kitap okudu adam" is also valid in Turkish (pragmatic change in the interpretation). This sentence can 
be analyzed with the same easiness if we are just looking for arguments of the predicate of the sentence.

As mentioned, noun phrases, denoting entities, are marked morphologically in Turkish to introduce their thematic roles, and this should be utilized in sentences like "adam kadına kitabı verdi" ("the man gave the book to the woman") since ontological constraints are not enough to analyze 'adam' ("man") as agent and 'kadın' ("woman") as goal (both are $H U M A N$ ). So, if there is only one event in a Turkish sentence and NPs are just single nouns, there is no need for a syntactic parsing to find the propositional content of the input sentence. The propositional content can be found using only knowledge from the ontology and thematic marks of nouns (like nominative, ablative, etc.). In this example, since 'adam' is in the nominative case and 'kadın' is in the dative case, 'adam' must be agent and 'kadın' must be goal of the GIVE predicate.

But, when the structures of NPs are complicated such as "masanin örtüsü" ("the cover of the table"), "üç kollu adam" ("the man with three arms"), "okul hakkında" ("about the school"), the naive approach above just simply fails. Since each NP denotes an entity in the sentence, a bracketing mechanism is needed to capture complex NPs. This is achieved through syntactic analysis based on a context-free grammar representing Turkish NPs [4]. Since our aim is to find the propositional structure, context-free rules are applied to propose syntactically correct $N P$ s denoting entities, and search for thematic arguments is done on these proposals. For example, consider the Turkish sentence "kadın kırmızı masa örtüsünü yıkadı" ("the woman washed the red table cover"). Syntactic component proposes the NP "kırmızı masa örtüsünü" ("the red table cover") as one entity, and then this proposed NP is attached as patient of $W A S H$.

$\begin{array}{lll}H U M A N & \longleftarrow & {[\text { kadın }]} \\ C O V E R(\text { is-for }(T A B L E), \text { color }(\text { red })) & \longleftarrow & {[\text { kırmızı masa örtüsünü] }} \\ W A S H(\text { agent }(H U M A N), \text { patient }(C O V E R)) & \longleftarrow & \text { [yıadı] }\end{array}$

Note that, this architecture forms a weak interaction between syntax and semantics (syntax proposes NP analyses as entities and search for thematic roles decides on their acceptance). In fact, ontology is not only utilized in finding argument structure of an event, but also used to check plausibility of proposed entities. Knowledge about the entity (head of NP) including its features and relations with other concepts is used to check whether proposed analysis can be correctly transformed to a concept instant (like COVER(is-for $(T A B L E)$, color $(r e d)$ ) given above). This usage of ontology provides an additional power in disambiguation. Let us reconsider the phrase "iki kolsuz adam" given in Section 2.

$\begin{array}{lll}\text { Cardinality }(\text { arm })=0 & {[\text { [iki kolsuz] adam] }} & \\ \text { Cardinality }=2 \& \text { Cardinality }(\text { arm })=0 & \longleftarrow & \text { kolsuz } \\ & \longleftarrow & \text { [iki kolsuz] }\end{array}$

Same mechanism also rejects the interpretation "my island with three arms" in "üç kollu adam" since the entity ISLAND has no feature to represent arm. 
Remember that, more informative analyses are preferred over less informative ones, and "üç kollu adam" is interpreted as "the man with three arms" since we have $H U M A N($ has-part $(A R M))$ and the analysis of "kollu adam" restates this fact.

Until here, only sentences with one verbal phrase are considered. When there are more than one verbal phrase, even the current architecture has some problems in finding the correct analysis since information about constituent ordering does not exist. For example, consider the following two sentences:

- "Kadın bardağı kıran çocuğa kızdı"

"The woman scolded the child who broke the glass"

- "Bardă̆ı kıran kadın çocuğa kızdı"

"The woman who broke the glass scolded the child"

The position change of the word 'kadın' ('woman') radically changes the interpretations of the sentences above, and this is not the kind of information that can be found in the ontology. It is the syntax that changes the interpretation, and it should be added somehow into the architecture. Like case marks in NPS denote the thematic roles of the entities, verbal suffixes present the semantic roles of the events in a sentence in Turkish [4. Main predicate is always distinguishable from the others and supplementary VPs are marked so that their roles can be found out. For example, $+E n$ suffix in Turkish (kıran $\leftarrow$ kır $+E n$ ) is used to express a definite description of an entity which is the agent of the event, and it requires the agent just after the verb. So, a verb with $+E n$ should be analyzed such that next NP is its agent and previous entities are to be checked whether they are arguments of the event. Thus, we get the following constituents for the first sentence, and the grouping for the supplementary event $B R E A K$.

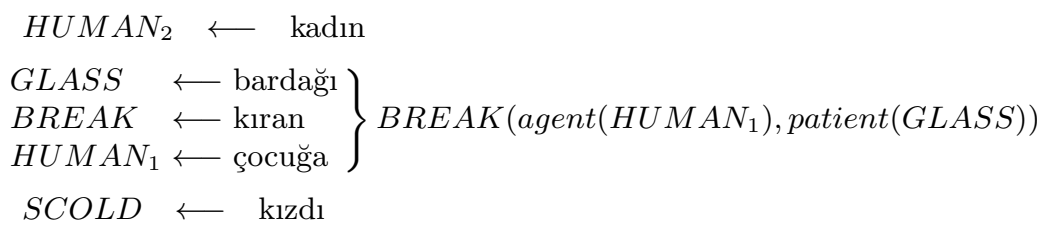

Since $B R E A K$ has only the arguments agent, patient, and instrument, and 'kadın' as a $H U M A N$ cannot be instrument, it cannot be treated as an argument of $B R E A K$. Since constructed proposition is for defining an entity, it is taken out from constituent list, only 'çocuk' (HUMAN 1 , a concept instance) is left as an entity. So, we have the following constituents and the grouping for the main event $S C O L D$ :

$$
\left.\begin{array}{l}
H U M A N_{2} \longleftarrow \text { kadın } \\
H U M A N_{1} \longleftarrow \text { çocuğa } \\
S C O L D \longleftarrow \text { kızdı }
\end{array}\right\} \operatorname{SCOLD}\left(\operatorname{agent}\left(H U M A N_{2}\right), \operatorname{goal}\left(H U M A N_{1}\right)\right.
$$

Note that, the two events of the example sentence have a common argument, $H U M A N_{1}$ ('çocuk'), that establishes the connection between the main and the supplementary event. The mechanism just described can be utilized 


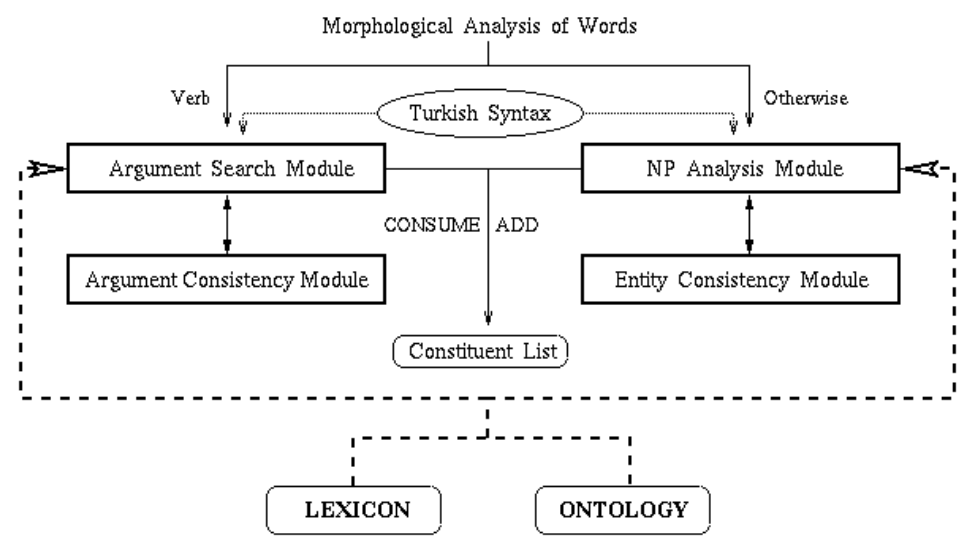

Fig. 1. Architecture of the Semantic Analysis

for other types of relations between events. For example, a verb with the suffix $+H_{\xi}$ in Turkish cannot have any other constituent after that verb, so its arguments should be searched in the previously encountered entities and its proposition should be added to the constituent list with erasing all its entities and itself. So, "Annemin Ankaraya gelişi beni çok sevindirdi" ("My mother's coming to Ankara made me very happy") is first transformed into analysis "COME $(\operatorname{agent}(H U M A N), \operatorname{goal}(C I T Y))$ beni çok sevindirdi", and then the final propositional analysis is achieved. This is the second place where syntax and semantics weakly interact.

As a conclusion, the computational architecture utilizes grammatical rules of Turkish without constructing a complete syntactic tree. Syntax is treated as a tool to help semantic disambiguation, and it proposes NP analyses for entities and constraints constituent ordering in cases of more than one event, expressed as VPs. But, it is the ontological constraints that decide on the propositional structure. Note that, search for arguments cannot interrupt the application of syntactic rules, it just rejects some proposed analysis. So, the proposed methodology conforms to weak interaction between syntax and semantics. Fig. 1 describes the overall computational architecture.

\subsection{TMR Construction}

After the TMR construction phase, the overall interpretation of the input sentence is represented in TMR formalism [26]. Since arguments of each event with their thematic roles are determined in the previous phase, only the existing semantic (aspect, modality, temporal relations, etc.) and pragmatic (speech-act, stylistics, etc.) properties of the sentence should be introduced into the constructed representation. But, before analyzing these additional information about the utterance, each found entity and event should be represented as a concept instance with a distinct frame. Beside the interpreted structure, each instance 
should take its definition from the lexicon (feature-value pairs associated with the used word sense) since concepts are proposed abstractions (not word senses). In other words, although 'man', 'woman', etc. are all HUMAN, they have different representations in the lexicon to constrain the set of individuals that can be referred with that word sense. Assuming that there is the word 'kadin' in an utterance, the following TMR frame is constructed.

\section{Lexicon Definition}

kadın

\begin{tabular}{lll} 
is-a & \multicolumn{1}{l}{ HUMAN } \\
definition & type & common \\
& gender & female \\
& age & $\geq 17$
\end{tabular}

\section{Constructed TMR Frame}

$\begin{array}{cl}H U M A N_{i} & \\ \text { type } & \text { common } \\ \text { gender } & \text { female } \\ \text { age } & \geq 17\end{array}$

After constructing the TMR frames for entities and events as a first step, semantic and pragmatic properties of the sentence are analyzed through maprules 11] which are content-based (whether a morpheme exists in a specific word, whether analyzed event is punctual or durative, whether constituents are in the default order, etc.) exclusive rules [7]. Each map-rule checks a set of constraints about morphology, syntax, and ontology, and it updates the TMR if those constraints are satisfied. In any TMR representation, temporal and epistemic properties of every event should be provided, and this is achieved by using suffixes of the verb and the event's aspectual properties defined in the ontology. The utilized rules look like the following:

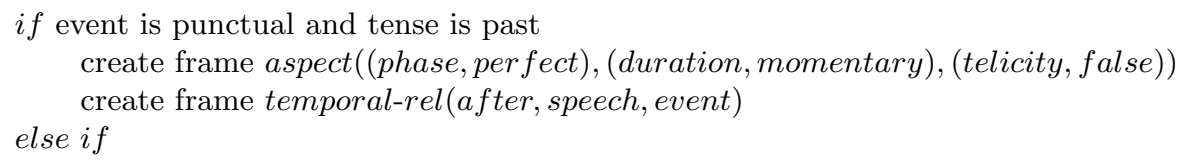

The information about speech-act is obtained from the main event (whether sentence is declarative or imperative, which constituent is the scope of the main predicate, etc.). Beside these, constituent order information is utilized to represent the speaker's attitude towards those components. If they are not in the default order, then constituents are attached pragmatic stance, topic, focus, and background. In addition, the change of speech focus in relative clauses and whtype questions is represented in speech-act frame.

\section{Conclusion}

The role of real world knowledge in comprehending natural language inputs is generally underestimated by the current computational architectures for $N L$ analysis. In this paper, we propose a new methodology for the analysis in which ontology, knowledge resource for representing real world, is the major resource that is utilized, instead of syntactic structure. We choose Turkish as the input 
language since its inflectional morphology provides enough information about the semantic properties of the components of a sentence. The proposed method is based on finding the argument structures of events using constraints on the thematic structures defined in the ontology.

First, we start with some observations about the language phenomenon which provides enough motivation about the need for an ontology in $N L$ analysis. We give examples in which syntactic constraints are not enough to eliminate irrelevant ambiguities. Then, we start with a naive approach which uses just ontological constraints, and new mechanisms are added successively such that our analysis becomes compatible with complex NPs and sentences in which there are more than one predicate. The final structure proposes a weak interaction between syntax and semantics, in which syntax just proposes analyses that guide the semantic disambiguation. If semantic analysis is successful, then computational model constructs a specific meaning representation, TMR, on top of the analyzed propositional structure.

\section{References}

1. J. R. Bateman. Ontology construction and natural language. In Proceedings of International Workshop on Formal Ontology, Pauda, Italy, 1993.

2. S. Beale, S. Nirenburg, and K. Mahesh. Semantic analysis in the mikrocosmos machine translation project. In Proceedings of the 2nd Symposium on Natural Language Processing (SNLP-95), Bangkok, Thailand, August 2-4, 1995.

3. B. J. Dorr. The use of lexical semantics in interlingua machine translation. Machine Translation, 4:3:135-193, 1993.

4. Z. Gungordu. A lexical-functional grammar for turkish. Master's thesis, Bilkent University, Ankara Turkey, July 1993.

5. K. Mahesh. Ontology development for machine translation: Ideology and methodology. In Memoranda in Computer and Cognitive Science MCCS-96-292, Las Crues, New Mexico State University, 1996.

6. K. Mahesh and S. Nirenburg. Meaning representation for knowledge sharing in practical machine translation. In Proceedings of the FLAIRS-96. Track on Information Interchange, Florida AI Research Symposium, Key West, Florida, May 19-22, 1996.

7. T. Mitamura and E. Nyberg. Hierarchical lexical structure and interpretive mapping in machine translation. In Proceedings of COLING-92, Nantes, France, July, 1992.

8. S. Nirenburg, J. Carbonell, M. Tomita, and K. Goodman. Machine Translation: A Knowledge-Based Approach. Morgan Kaufmann, San Mateo, California, 1992.

9. K. Oflazer. Two-level description of turkish morphology. Literary and Linguistic Computing, 9:2, 1994.

10. M. Steedman. Computational aspects of the theory of grammar. An Invitation to Cognative Science, Language, pages 247-283, 1995.

11. M. Temizsoy. Design and implementation of a system for mapping text meaning representations to f-structures of turkish sentences. Master's thesis, Bilkent University, Ankara Turkey, August 1997. 
12. M. Temizsoy and I. Cicekli. A language-independent system for generating feature structures from interlingua representations. In Proceedings of the 9th International Workshop on Natural Language Generation, Niagara-on-the-Lake, Canada, August, 1998. 